\title{
Niveles de desarrollo de los programas de formación en habilidades informativas- alfabetización informacional en universidades mexicanas según la información de sus sitios Web*
}

\author{
Alejandro Uribe Tirado ${ }^{\star \star}$
}

Artículo recibido:

22 de marzo de 2012.

Artículo aceptado:

9 de agosto de 2012.

\section{RESUMEN}

El desarrollo de habilidades informativas (DHI) o alfabetización informacional (ALFIN), entendida como la formación en competencias informacionales, es uno de los retos actuales de las bibliotecas universitarias ante las posibilidades de acceso a enormes recursos de información que facilitan los medios digitales al interior y exterior de las bibliotecas, y la necesidad de sus usuarios de formarse en mejores estrategias y herramientas para acceder, seleccionar y evaluar la información más pertinente y de mayor calidad. Ante esta situación las bibliotecas universitarias iberoamericanas han ido poco

* $\quad$ Este texto se enmarca en la investigación: "Lecciones aprendidas en programas de Alfabetización Informacional en universidades de Iberoamérica" (Uribe-Tirado, A. $2010-\ldots)$.

** Universidad de Antioquia, Escuela Interamericana de Bibliotecología, Colombia. auribe@bibliotecologia.udea.edu.co

INVESTIGACIÓN BIBLIOTECOLÓGICA, Vol. 26, Núm. 58, septiembre/diciembre, 2012, México, ISSN: 0187-358X. pp. 121-151 
a poco incorporando esta formación, bien sea desde programas/cursos directos o mediante el trabajo colaborativo con docentes y facultades en currículas de toda una universidad o de disciplinas específicas. Este trabajo, desde la metodología de análisis de contenidos Web, revisa la información que presentan las bibliotecas universitarias mexicanas sobre esta actividad de formación para, a partir de la misma, visualizar los niveles de desarrollo que tendrían de sus programas de DHI-ALFIN, y por consiguiente, las competencias informacionales que estarían adquiriendo sus usuarios.

Palabras Clave: Alfabetización informacional; Desarrollo de habilidades informativas; Competencias informacionales; Bibliotecas universitarias; México; Análisis de contenidos; Sitios web.

\section{ABSTRACT}

Levels of development of information literacy in Mexican universities as gauged by an examination of training programs posted on university websites Alejandro Uribe Tirado

Information literacy, or information skills, understood as information competency training, is a key challenge of university libraries as it relates to the ability provided by digital media to access vast information resources and the user public's need to understand and apply best practices in selection and evaluation of information in order to retrieve the most relevant and highest quality information. This study shows that Ibero-American university libraries (Latin-America, Spain and Portugal) have been gradually incorporating training such through the library itself or through collaborative curricula approaches. By examining Mexican university library website contents on core information training, this study assesses the level of information literacy and competency training offered by each university.

Keywords: Information literacy; Information competencies; University libraries; México; Content analysis; Web sites. 


\section{INTRODUCCIÓN}

T a DHI-ALFIN es una temática que desde mediados de los años ochenta 1 del Siglo XX, con sus debidas precisiones y adaptaciones sobre su alcance y sus aspectos terminológicos, conceptuales y teóricos, ha venido desarrollándose en Iberoamérica, ${ }^{1}$ y de manera particular en México, ${ }^{2}$ en el contexto de las bibliotecas, especialmente aquéllas de nivel universitario que es donde, en términos generales, ha habido más avances, y en donde se marcan las futuras tendencias para las otras tipologías de bibliotecas: escolares, públicas, nacionales y especializadas, (Pinto-Molina y Uribe-Tirado, 2011).

Tras más de 25 años de desarrollos en esta temática, de precisiones y adaptaciones que han llevado de la instrucción bibliográfica y la formación de usuarios tradicional hasta llegar hoy al paradigma de la formación en DHI-ALFIN (Gómez Hernández y Benito Morales, 2001; Gómez-Hernández, 2007; Pinto-Molina, Sales y Osorio, 2008; etc.), se han dado diferentes procesos de avance, periodos de desarrollo (Uribe-Tirado, 2010a-b):

Pre-Inicio (1985-1994); Inicio (1995-1999); Pre-Avance (2000-2003); Avance (2004-2007) y Pre-Posicionamiento (2008 - ...), además de la visualización de un $6^{\circ}$ periodo denominado: Posicionamiento (a partir del 2012 o 2013 ?).

Ante esos procesos un aspecto clave es identificar cómo se está llevando a cabo en la educación superior, tanto en las universidades como en las demás instituciones de educación superior -IES- en el contexto iberoamericano, la incorporación de esta formación en competencias informacionales, para así identificar las lecciones aprendidas y buenas prácticas que les permitan a otras bibliotecas universitarias, que están iniciando el desarrollo de programas de DHI-ALFIN, poder mejorar y avanzar en sus programas formativos tras hacer las debidas adaptaciones.

Para dicha identificación una fuente de información clave son los sitios Web de las bibliotecas, ya que especialmente desde la última década, es este medio sin duda el que más impacto está teniendo en la divulgación de estos servicios y es, por lo tanto, objeto de continua investigación (Detlor y Lewis, 2006; Gardner, Juricek y Xu, 2008, entre otros).

1 Ver Wiki-Reposotorio ALFIN / Iberoamerica, http://alfiniberoamerica.wikispaces.com (consultado: 8-08-2012).

2 Ver por ejemplo, la lista: DHI-UACJ / Desarrollo de Habilidades Informativas: http://mx.groups. yahoo.com/group/dhi-uacj/?v=1\&t=search \&ch=web \&pub=groups \&sec=group \&slk=1, que incluye gran parte de lo que se ha ido desarrollando en México en esta temática desde el año 2004, y la sesión específica para México del Wiki-Repositorio ALFIN / Iberoamérica: http://alfiniberoamerica.wikispaces.com/México (consultado: 8-08-2012). 
Esto se aprecia específicamente en los servicios de formación (Ferreira Gomes, Prudêncio y da Conceiçã, 2010), y en la divulgación e interacción que permiten los sitios Web (sea desde la Web 1.0 ó 2.0) tanto para los públicos internos que quieren aprovechar su oferta formativa (estudiantes, profesores, investigadores y/o directivos de su institución a quienes van dirigidos sus cursos o tutoriales de formación en DHI-ALFIN), como para los externos (bibliotecólogos, bibliotecarios, profesores, directivos de otras universidades/IES) que quieren conocer la oferta formativa de una determinada institución y biblioteca para realizar procesos de benchmarking, de trabajo cooperativo interinstitucional, de consorcios formativos, etcétera.

Este trabajo presenta un análisis del caso particular de México, ${ }^{3}$ país donde -según la revisión bibliográfica realizada previamente (Anexo 1) todavía no se ha hecho un análisis similar, ya que un paso importante para avanzar en esta temática, como en otras problemáticas de carácter social, es contar con un diagnóstico que dé cuenta de la situación, para establecer líneas de acción destinadas a entidades nacionales o universitarias que coordinen el accionar de las bibliotecas. Desafortunadamente en el contexto latinoamericano ese trabajo estaba pendiente desde hace varios años, como se evidenció en el documento sobre el Estado del Arte de la Alfabetización Informacional realizado con el apoyo de la International Federation of Libraries Association-IFLA- (Lau et al, 2006).

Nuestro objetivo por tanto ha sido, luego de realizar el análisis de los sitios web de las bibliotecas de sus universidades o IES y de la información que presentan sobre el desarrollo de programas de DHI-ALFIN, visualizar los posibles aportes que desde esas bibliotecas universitarias mexicanas se estaría haciendo a la incorporación de la formación en competencias informacionales (DHI-ALFIN), desde una perspectiva de universidad/IES alfabetizadas informacionalmente (Webber y Johnston, 2006) y de usuarios competentes en información (ALA-ACRL, 2000).

\section{MARCo TEÓRICO-CONCEPTUAL Y METODOLÓGICO}

Tomando en cuenta postulados desarrollados en trabajos anteriores (UribeTirado, 2010), que integran aportes de diferentes autores, y haciendo una generalización de los distintos contextos, se puede decir que una universidad/IES o dependencia dentro de la misma institución que lidere procesos

3 Textos semejantes con el análisis particular para otros países iberoamericanos están también en proceso de evaluación, edición y/o publicación en Colombia, Cuba, Portugal, Puerto Rico, Brasil, España, Argentina, Chile, Perú, Costa Rica etcétera. 
o programas de alfabetización informacional (tales como un Sistema de Bibliotecas, Biblioteca, Centro de Información, CRAI, etc.), puede clasificarse en cuatro categorías según el grado en que sus programas de DHI-ALFIN se hayan implementado, y por lo tanto, según la adquisición de las competencias informacionales que estarían teniendo sus usuarios:

1) Universidades/dependencias comprometidas en la formación en DHI-ALFIN:

Se caracterizan generalmente por un trabajo en DHI-ALFIN en el que las competencias informacionales son definidas tanto en objetivos como en metas concretas en sus planes estratégicos, como consecuencia de la concientización sobre su importancia para el aprendizaje para toda la vida y la generación de conocimientos, apoyados en las TIC, el e-learning y en estratégicas didácticas activas, frente a las exigencias educacionales e informacionales de la actual sociedad. Por ende reconocen la necesidad de que las distintas poblaciones de su comunidad (estudiantes, profesores, investigadores, empleados, directivas) adquieran esas competencias y tengan diferentes opciones de formación. Opciones de formación de carácter curricular para el caso de los estudiantes, y de educación continua y como requisito de vinculaciónactualización-ascenso en los escalafones para el caso de profesores, investigadores, empleados y directivos. Los programas y cursos que conforman estas opciones de formación en DHI-ALFIN presentan, a su vez, objetivos y metas de enseñanza-aprendizaje definidos, la adopción de un modelo, norma o estándar de DHI-ALFIN, además de una evaluación permanente de sus resultados (tanto cuantitativos como cualitativos) y un mejoramiento continuo. Las áreas a cargo de los programas o cursos que hacen operativa esta formación trabajan en forma colaborativa o integrada, y reciben todo el apoyo institucional en recursos financieros y tecnológicos y en capital humano, lo cual les permite optimizar esfuerzos y recursos, por lo que los programas/cursos presentan las mejores y más actualizadas posibilidades de acceso, conocimiento y uso de herramientas y servicios especializados de Internet y otras fuentes de información de calidad.

2) Universidades/Dependencias en crecimiento en la formación en DHI-ALFIN:

Son las que incluyen en sus planes estratégicos la alfabetización informacional y los objetivos y metas que esto implica; sus diferentes componentes se están apenas formalizando para ser considerados como claves para su desarrollo institucional. Se comienza con ellos a tener 
conciencia sobre la transversalidad de la DHI-ALFIN en la formación y capacitación de las distintas poblaciones que conforman la comunidad universitaria, pero aún hay muchas instancias en su interior que no tienen claras sus implicaciones cognitivas, didácticas, informacionales, tecnológicas y comunicativas. Los cursos/programas de DHI-ALFIN que ya llevan a cabo están en proceso de estructuración, definiendo sus objetivos y metas de aprendizaje, y acercándose a la concreción y aplicación de un modelo, norma o estándar de DHI-ALFIN, aunque con pocas experiencias mediante e-learning y didácticas activas. Estos conocimientos comienzan a ser aceptados como parte de los planes curriculares sólo en algunas Facultades, y como necesidades de vinculación para los nuevos profesores, investigadores, empleados y directivos. Las evaluaciones de los programas/cursos que se llevan a cabo en forma más permanente se centran en lo cuantitativo, aunque ya con algunos avances cualitativos, y no todas las veces generan mejoramientos las valoraciones hechas por los participantes. Cada vez se cuenta con mayores recursos financieros, tecnológicos y de capital humano, pero son todavía insuficientes para las necesidades y requerimientos que las poblaciones universitarias les exigen a las áreas encargadas. Las posibilidades de acceso, conocimiento y uso de herramientas y servicios especializados de Internet y otras fuentes de información de calidad aumentan cada vez más pero aún no alcanzan para lograr mejores resultados.

3) Universidades/Dependencias que están iniciando la formación en DHIALFIN:

Éstas son las que han comenzado desde hace 1 o 2 años a reconocer que los programas tradicionales de formación de usuarios (entrenamiento para el uso de los servicios de la biblioteca) no son suficientes, y que la gestión de información y del conocimiento implica, en la actualidad, el aprendizaje para toda la vida y mayores alcances en esa formación, para utilizar las grandes posibilidades de los medios digitales y nuevas formas de enseñanza-aprendizaje (e-learning y didácticas activas). Por lo tanto, la DHI-ALFIN no aparece en forma definida y formal, entre sus objetivos y metas estratégicas, y se conciben más como cursos aislados o complementarios no curriculares o de exigencia en adquisición de competencias. Los nuevos cursos de DHIALFIN se van construyendo sobre la base de los cursos o programas de formación de usuarios tradicionales, pero poco a poco se comienzan a adicionar o reestructurar pensando en las implicaciones de DHIALFIN, tanto filosófica como operativamente, y su evaluación se hace 
presente en forma esporádica para ser parte de la divulgación de distintos informes desde una perspectiva cuantitativa. Los recursos con que cuenta son pocos y se está más en la tarea de generar experiencias piloto para, por medio de sus resultados positivos, ir gestionando nuevos recursos tanto financieros y tecnológicos como de capital humano y de fuentes de información de calidad.

4) Universidades/Dependencias desconocedoras de la formación en DHIALFIN:

Son aquéllas donde el tema de la alfabetización informacional, como concepto, no aparece o se confunde con la alfabetización digital, considerándose que con ésta última es suficiente, y que si se tiene acceso a los medios digitales, los procesos de aprendizaje permanente y generación de conocimientos se darán por sí solos. Así, cuando en sus planes estratégicos se menciona algún aspecto es con enfoque en las tecnologías en Internet como fin, más que como medio. De igual manera cuentan con programas tradicionales de formación de usuarios que la mayoría de las veces son muy precarios, lo que responde a una concepción muy pobre de lo que implican una biblioteca o fuentes de información de calidad para mejor procesar la enseñanza-aprendizaje, por lo que sus recursos financieros y tecnológicos, de capital humano y de fuentes de información de calidad son casi nulos. Dichos programas no tienen metas y objetivos muy definidos y son más bien producto de la inercia de estar realizando lo mismo desde hace varios años, bajo la concepción acostumbrada de formación de usuarios. Son instituciones educativas que tienen una tradición más acorde con las universidades del siglo XX que con las exigencias de la actual sociedad de la información.

En síntesis estas categorías han tenido en cuenta para su descripción lo que implica la DHI-ALFIN como paradigma de formación (Pinto, Sales y Osorio, 2008) y la evolución que representa frente a la formación tradicional de usuarios (Gómez Hernández y Benito Morales, 2001; Gómez Hernández, 2007), pueden identificarse al analizar los sitios Web de las bibliotecas de las universidades-IES, y procurar detectar el posible nivel de incorporación de esta formación -explorando la sección o la información concreta sobre servicios de formación de la biblioteca-, en diferentes elementos (variables), en tanto que presentan los siguientes elementos:

1) Definición del programa: misión, visión, objetivos, alcance, plan operativo, plan pedagógico. 
2) Fundamentación del programa: definición de formación (como formación de usuarios -F.U.- o como DHI-ALFIN), modelo pedagógico-didáctico y estándar-indicadores asumidos.

3) Estructuración del programa: cursos ofrecidos, modalidad de los cursos, incorporación de las TIC, Web 2.0 y de ambientes virtuales de aprendizaje, presencia curricular o extracurricular de los cursos.

4) Evaluación del programa: indicadores de gestión, indicadores de resultados de aprendizaje, procesos de mejoramiento continuo.

Adicionalmente se observa también la presencia de información complementaria, como:

5) Publicaciones sobre este tema y la experiencia de la biblioteca y del programa de formación: artículos, ponencias, recursos Web 2.0.

6) Participación en grupos y redes de trabajo sobre esta temática: redes de bibliotecas universitarias, colectivos de bibliotecólogos/bibliotecarios, etcétera.

7) Participación en medios y recursos de divulgación y aprendizaje de esta temática: listas de discusión, blogs, wikis, twitters, boletines, comunidades virtuales, etcétera.

De estas 7 variables es especialmente la referente a Estructuración del programa la que puede dar más cuenta del grado de incorporación que ALFIN tendría en una Universidad-IES y su Dependencia a cargo (BibliotecaCRAI, etc.), ya que la presencia de determinados Cursos-Tutoriales permite visualizar con mayor facilidad el alcance que tiene el programa (facultades, titulaciones, número de estudiantes, etc.) y si se trabajan las 5, 6 o 7 competencias que presentan los modelos-estándares universitarios de ALFIN (según el que se haya elegido seguir: ALA-ACRL, CILIP, ANZIIL, DHI Ciudad Juárez, entre otros). ${ }^{4}$

Esto conlleva a una correlación de esta variable específica, que se puede visualizar desde los sitios Web, con las 4 categorías de incorporación que dan cuenta del proceso de desarrollo de esta temática, la cual ha sido avalada por expertos internacionales (Sonntag, 2011) y comparte la visión de documentos y propuestas que tiene EMPATIC de la Unión Europea (2009), que cita al marco Australiano-Neozelandés —ANZIL—, antes de dar el paso al interior de las Universidad-IES de la instrucción bibliográfica y a la formación de usuarios tradicional hasta llegar hoy a la ALFIN en lo curricular: 
- Instituciones comprometidas:

- DHI-ALFIN Nivel 2: la biblioteca ofrece directamente cursos para formar en competencias informacionales que incorporan lo instrumental + aprendizaje para toda la vida + pensamiento crítico, y cursos o módulos específicos inmersos oficialmente en la currícula, distintos programas académicos/carreras para formar de manera transversal y disciplinar en esas competencias.

- Instituciones en crecimiento:

- DHI-ALFIN Nivel 1: la biblioteca ofrece directamente cursos para formar en competencias informacionales que incorporan lo instrumental + aprendizaje para toda la vida + pensamiento crítico.

- Instituciones que se inician:

- Formación de Usuarios. Nivel 1: la biblioteca ofrece directamente capacitación en servicios generales de la biblioteca y algunos cursos -muy instrumentales- para búsqueda de información: utilización de catálogos/bases de datos, aunque se comienza a analizar la necesidad de cambiar esta formación tradicional y de trabajar las demás competencias.

- Instituciones desconocedoras:

- Formación de Usuarios. Nivel 2: la biblioteca ofrece directamente sólo capacitación para el uso del catálogo.

- No hay presencia de ningún tipo de formación-capacitación

Es importante indicar al tener en cuenta este marco metodológico y teórico-conceptual para visualizar los posibles niveles de desarrollo de DHI-ALFIN en las bibliotecas universitarias, que esta categorización es una aproximación a partir de la información presente en los sitios Web, pero en ningún momento quiere esto decir que identificar a una u otra universidad-IES/dependencia en alguno de estos cuatro tipos implique su total realidad en esta temática, ya que no siempre se publica o informa en los sitios web todo lo que se hace; o por el contrario, a veces se publica o informa más de lo que realmente se hace. Es decir, este tipo de análisis se hace en un momento y desde un ángulo determinado, que lleva a una aproximación y sistematización útil como visión general del desarrollo que se daría en la formación en DHI-ALFIN.

\section{Diseño METODOLÓGICO}

Para recopilar la información de las bibliotecas de las universidades/IES de México se eligió como fuente inicial de identificación de los sitios Web de dichas 
instituciones al sitio del Directorio Nacional de Instituciones de Educación Superior. ${ }^{5}$ A su vez, con el fin de adicionar y depurar ese listado se recurrió al Ranking Web de Universidades del Mundo del CCHS/CSIC ${ }^{6}$ y al Portal de los Estudiantes El Altillo.Com? ${ }^{7}$

La integración de estas tres fuentes generó un listado inicial de 1634 instituciones. Sobre este listado se hizo una confirmación de cuántas de ellas estaban en distintos estados y ciudades mexicanas aunque pertenecían a una misma organización y se trataba de sedes diferentes. Su sitio Web era el mismo para todas (un mismo url) y también se confirmó cuáles tenían url activos y con información vigente (actualizada en el último año).

Tras esa depuración, finalmente se generó un listado de 532 universidades/IES mexicanas: 260 instituciones públicas y 272 privadas (Anexo 2). ${ }^{8}$

Para realizar el análisis de cada una de ellas se utilizó una ficha de registro, considerando postulados del análisis de contenidos (White y Marsh, 2006), en la que se consignó la información básica de cada biblioteca, su url y la información sobre DHI-ALFIN teniendo en cuenta las posibles 7 variables a valorar, pero enfatizando especialmente la estructuración del programa. Esa información volcada en una base de datos permitió identificar, mediante análisis tipológico, en cuál de los cuatro niveles de desarrollo se encontraría cada biblioteca universitaria.

\section{Resultados}

Tras el análisis de estas 532 bibliotecas universitarias mexicanas según la información de sus sitios Web, se obtuvieron los resultados que se sintetizan en las siguientes figuras.

5 Disponible en: http://sistemas.anuies.mx/perl/directorios/selrec1.pl, (consultado: 20-2-2012).

6 Disponible en: http://www.webometrics.info/university_by_country.asp? country=mx (consultado: 20-2-2012).

7 Disponible en: http://www.altillo.com/universidades/universidades_mex.asp (consultado: 20-2-2012).

8 Ver y descargar este anexo en: https://docs.google.com/open?id=0BweUYcipCswRR1BKX1JERj Zmdm8 (consultado: 8-08-2012). 


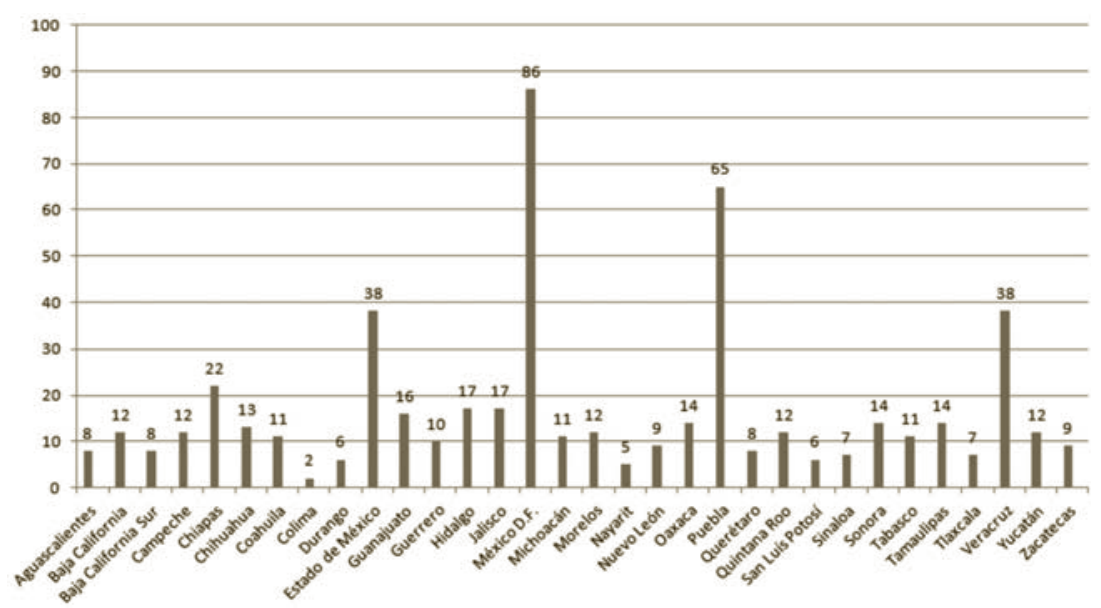

Figura 1. Núm. de bibliotecas universitarias mexicanas analizadas según estados.

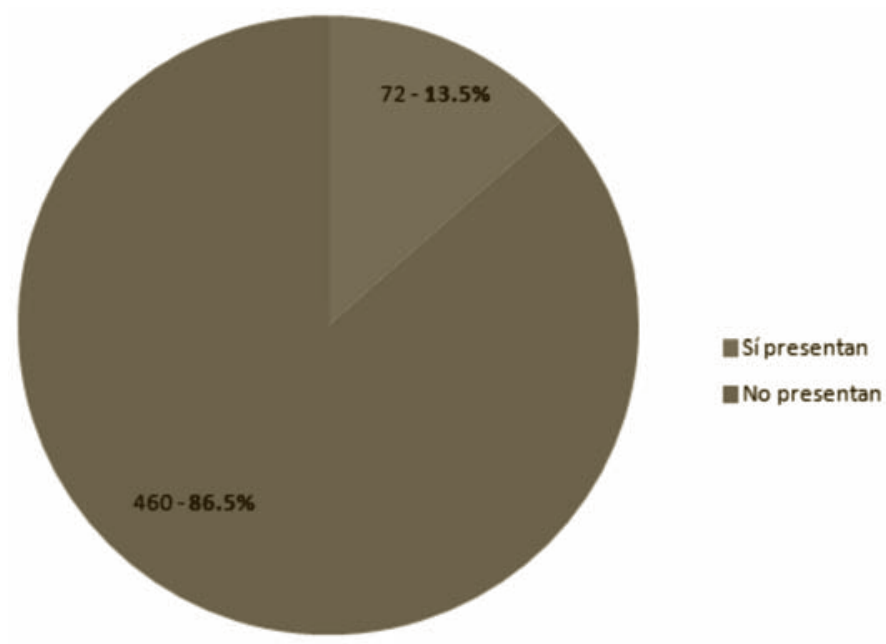

Figura 2. Núm. de bibliotecas universitarias mexicanas que presentan información sobre algún nivel de formación (F.U.: Formación de usuarios tradicional - DHI-ALFIN). 


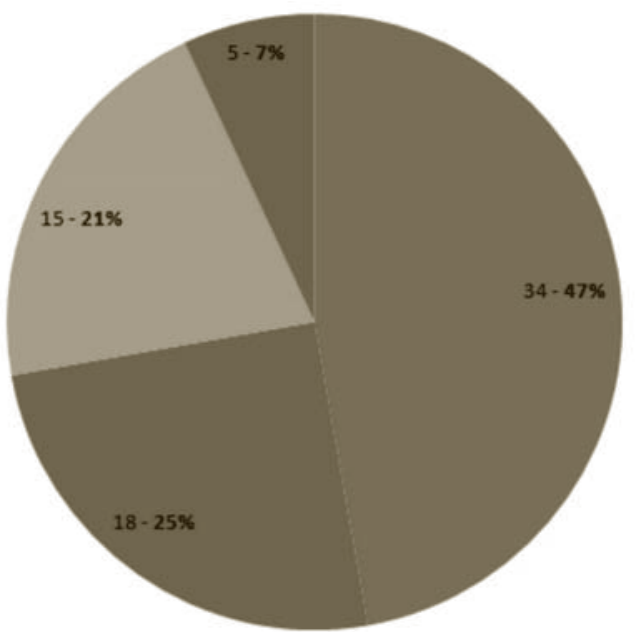

- Formación de usuarios. Nivel 2

E Formación de usuarios. Nivel 1 = ALFIN/DHI. Nivel 1

aLFIN/DHI. Nivel 2

Figura 3. Tipología de universidades-IES mexicanas que presentan información según los niveles de desarrollo de sus programas de formación (F.U. o DHI-ALFIN).

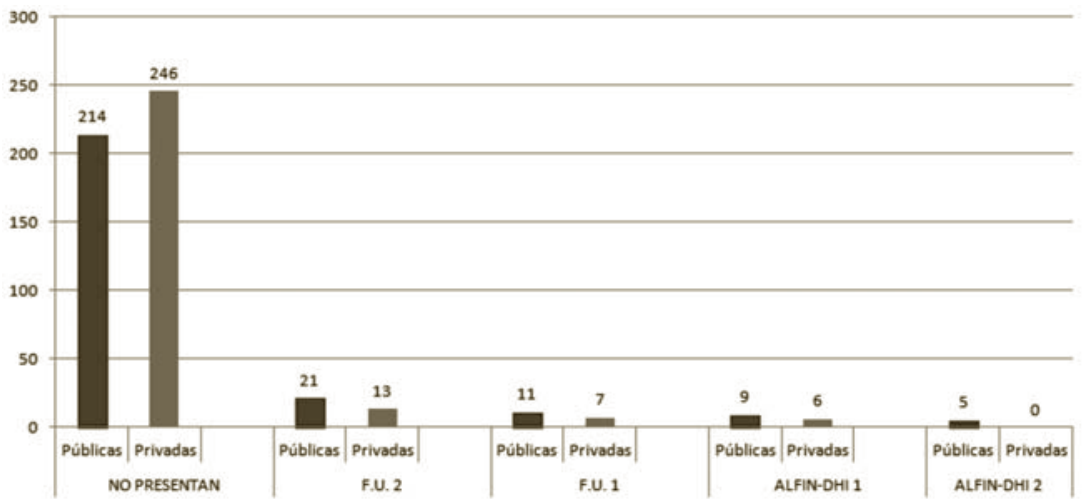

Figura 4. Niveles de incorporación F.U. o DHI-ALFIN de las bibliotecas universitarias mexicanas según su tipología de públicas o privadas. 

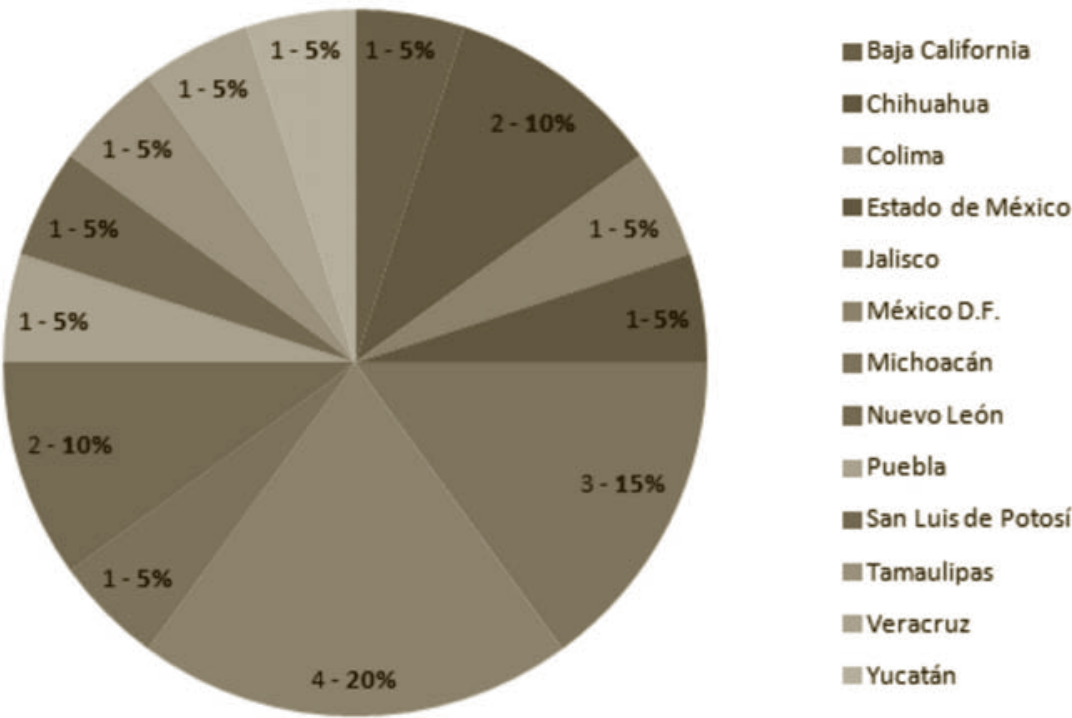

Figura 5. Procedencia de las bibliotecas universitarias mexicanas que presentan niveles 102 de DHI-ALFIN.

\section{AnÁlisis y Discusión}

Para iniciar este apartado es necesario indicar que para el análisis realizado y la interpretación de los resultados partimos de dos postulados:

- La alfabetización informacional o desarrollo de habilidades informativas es una función constitutiva del papel que deben cumplir las bibliotecas universitarias frente a los retos de la educación superior de hoy, considerando los requerimientos de la Sociedad de la Información.

- La información que se publica en la Web, ante la generación de usuarios .Net, Google, 2.0, o como se les quiera denominar, es un aspecto fundamental para que todos los servicios de las bibliotecas se conozcan y su posicionamiento aumente como mediadora de la información con valores agregados, y por tanto, si este medio no se gestiona de forma continua se estará perdiendo una gran posibilidad de servicio para los usuarios, que son la razón de ser de toda biblioteca.

Considerando lo anterior al analizar los resultados, podemos decir que: 
Este estudio evidencia (Figura 2) que en el caso de las bibliotecas universitarias mexicanas hay un número-porcentaje muy alto de instituciones tanto públicas como privadas (460 equivalentes al $86.5 \%$ del total analizadas: 214 públicas y 246 privadas) en las que ambos postulados o uno de ellos no se estaría cumpliendo, lo cual debe ser un llamado de atención para estas instituciones teniendo en cuenta los efectos en la calidad de la educación que tiene la alfabetización informacional (Área-Moreira, 2007).

Es decir que:

- Estas instituciones (desde sus bibliotecas) no están realizando ningún o casi ningún tipo de actividad de formación (en algún nivel de F.U. o de DHI-ALFIN), o que sí, lo están haciendo.

- Falta trabajar en la divulgación y marketing de este servicio, que es clave para las bibliotecas universitarias del siglo XXI y su posicionamiento entre los usuarios, las directivas universitarias y la sociedad en general (Webber y Johnston, 2006).

A su vez, en una proporción importante de las que informan algo sobre programas de formación (72 instituciones equivalentes al 13.5\% del total analizadas), esta formación se centra aún (Figura 3) en los niveles F.U. 2 o 1 (34 universidades/IES en F.U. nivel 2 y 18 en F.U. nivel 1, equivalentes sumando ambos niveles al $72 \%$ ), que se refieren más a la formación tradicional y a la inercia en el trabajo formativo que durante años han realizado las bibliotecas, que a los requerimientos que la sociedad actual está exigiendo -como lo reitera la UNESCO desde sus Declaraciones $(2003,2005,2011)$ y sus continuas publicaciones ${ }^{9}$-considerando la formación en competencias informacionales como una de las bases del aprendizaje crítico y para toda la vida.

Cuando se indaga sobre el carácter de universidad/IES pública o privada (Figura 4) y los niveles de incorporación, llama la atención, en primer lugar, que hay una relativa paridad entre las universidad/IES públicas y las privadas que no presentan información sobre formación (sea desde niveles de F.U. o de DHI-ALFIN). Pero cuando se profundiza en los resultados obtenidos desde el análisis de contenidos Web, preocupa el hecho de que entre las 460 universidades/IES que no presentan información sobre formación, en 207 de ellas ni siquiera hay en sus sitios Web un área o sección que hable de la biblioteca,

9 Disponible en: http://portal.unesco.org/ci/en/ev.php-URL_ID=27302\&URL_DO=DO_TOPIC\&URL_ SECTION=-465.html [15-12-2011]. 
centro de información, etcétera. (equivalente al 45\% de éstas, y al 39\% del total de las 532 universidades/IES analizadas en este trabajo), lo que suscita las siguientes preguntas: ¿cómo se concibe una entidad educativa sin biblioteca? ¿Qué lugar de importancia académica e investigativa se le da a la biblioteca si ni siquiera se resalta o anuncia en los sitios Web de la respectiva universidad /IES?

A su vez, entre las restantes 253 universidades/IES que sí tienen una sesión o área en sus sitios Web universitarios relativos a la biblioteca, en 95 casos la sección o área Web de biblioteca es un espacio de links a bases de datos o a sitios de otras bibliotecas universitarias, lo cual también conduce a las preguntas: tomando en cuenta los avances del mundo digital, ¿es posible pensar en nuestras universidades/IES que la biblioteca sea sólo digital o virtual? ¿Es una biblioteca digital o virtual un sitio Web con links a bases de datos?

Lo anterior es un análisis resumido en lo referente a las universidades/IES que no presentaron información sobre formación, pero entre las que sí la presentaron podemos indicar, de manera también sintética, que se le da mayor predominio a la formación como un servicio clave de las bibliotecas entre las de carácter público que entre las de carácter privado, y sobre todo cuando se consideran las universidades/IES que han logrado que esta formación tenga un reconocimiento curricular (DHI-ALFIN 2), entre las que solo ubicamos universidades/IES de carácter público.

Adicionalmente, y para terminar este apartado resumido, ${ }^{10}$ es importante reiterar que de las 20 bibliotecas universitarias que estarían en los niveles 1 o 2 de ALFIN, éstas se encuentran concentradas (figuras 1 y 5) en determinados estados y en el Distrito Federal (México DF), lo cual implica por un lado mayores posibilidades de trabajo conjunto entre estas universidades/IES (12 Estados y el DF, equivalentes al 40\% del total de estados mexicanos) para seguir avanzando en DHI-ALFIN y poder optimizar recursos físicos, compartir contenidos, generar redes y crecer en el capital humano, como se está haciendo ya en otros contextos iberoamericanos. ${ }^{11}$ Pero por el otro surge una preocupación y un reto sobre cómo extender estos desarrollos a universidades-IES de México ubicadas en otras regiones (los restantes 19 estados) donde no se ubicaron universidades/IES con bibliotecas que ofrezcan servicios de formación en niveles 1 ó 2 de DHI-ALFIN, para que así haya niveles equitativos

10 Afirmamos que en forma resumida, ya que cada dato da para profundizar más y más, considerando las posibilidades de diferentes cruces de variables, pero esto iría más allá del carácter de diagnóstico general que pretende presentar este texto.

11 Proyecto Unici2 España: http://www.alfared.org/blog/bibliotecas-universitarias/1212 (consultado: 15-12-2011]. 
de formación en competencias informacionales para todas las comunidades universitarias mexicanas, independientemente del Estado y la ciudad.

\section{CONCLUSIÓN GENERAL}

En síntesis este estudio evidencia que en el caso de México hay importantes desarrollos en algunas universidades-IES, ${ }^{12}$ pero que una gran cantidad de ellas no tiene todavía programas, ni tienen desarrollos importantes (niveles F.U. 2 o 1), o no los divulgan desde sus sitios Web, y menos aún desde la literatura académica y científica del área, considerando el trabajo de análisis documental hecho paralelamente a este análisis de contenido Web (desde el Wiki ALFIN Iberoamérica, sección México) como otro proceso de la investigación macro que abarca el presente trabajo (ver nota al pie marcada con * al principio del presente artículo).

Por tanto se puede concluir que queda mucho trabajo por hacer en toda biblioteca para su universidad/IES, y desde todas las bibliotecas mexicanas para asumir la DHI-ALFIN como un proceso -y a la vez una estrategia- fundamental y transversal. Se trata de un reto no sólo para las bibliotecas sino también para las áreas de sistemas o de cómputo de las mismas para trabajar de manera más colaborativa tanto en lo referente a la gestión de contenidos de los sitios web de las bibliotecas ("lo que no se muestra no se conoce y valora"), como en cuanto a trabajar las competencias que el mundo de hoy exige y que se reconocen como complementarias: competencias informáticas e informacionales (Area Moreira, Gros y Marzal, 2008; REBIUN-CRUE, 2009; Gutiérrez, Vidal, y Area-Moreira, 2012).

Estos son retos para México y otros países iberoamericanos con similares niveles de desarrollo en DHI-ALFIN: ${ }^{13}$ avanzar buscando cada vez un trabajo más colaborativo entre las bibliotecas universitarias de los propios países y las del exterior, las áreas de cómputo-sistemas, y las vicerrectorías de docencia-académicas e investigación.

No obstante en ese sentido de trabajo colaborativo y mayor divulgación y posicionamiento es necesario destacar tres aspectos particulares del contexto mexicano:

12 Ver listado de universidades mexicanas destacadas: http://g.co/maps/4tmva, (consultado: 8 08-2012).

13 Ver informe: "La incorporación de ALFIN como servicio fundamental de las bibliotecas Iberoamericanas. Una mirada general desde la construcción de un mapa de geo-representación de contenidos" (Uribe-Tirado, 2012), disponible en el especial de ALFARED: http://www. alfared.org/content/veintitantas-experiencias-alfin-y-una-canci-n-esperanzada/los-b-sicosde-alfin/la-incorporac (consultado: 15-01-2012). 
Cabe destacar el trabajo que desde la Universidad Autónoma de Ciudad Juárez se viene haciendo desde 1997 para tratar de impulsar (no sólo a México sino a toda Iberoamérica), con la realización periódica del Encuentro Internacional DHI, que va, hasta el momento, en su séptima versión:

- Primer encuentro internacional DHI, Tema: "Desarrollo de Habilidades Informativas en Instituciones de Educación Superior”. 1997, http://bivir.uacj.mx/dhi/Eventos/Default.htm.

- Segundo encuentro internacional DHI, Tema: "La Instrucción de Usuarios ante los Nuevos Modelos Educativos" 1999, http://bivir.uacj. $\mathrm{mx} / \mathrm{dhi} /$ Eventos/Default.htm.

- Tercer encuentro internacional DHI, Tema: "Normas para Alfabetización Informativa” 2002, http://bivir.uacj.mx/dhi/Eventos/Default. htm.

- Cuarto Encuentro Internacional DHI, Tema: "Competencias Informativas: Hacia la implementación de Programas”. 2004, http://bivir.uacj. $\mathrm{mx} / \mathrm{dhi} /$ Eventos/Default.htm.

- Quinto Encuentro Internacional DHI, Tema: "Diagnóstico y Evaluación: Elementos Fundamentales para los Programas de Alfabetización Informativa" 2006, http://bivir.uacj.mx/dhi/Eventos/Default. htm.

- Sexto Encuentro Internacional DHI, Tema: "La innovación educativa en programas de desarrollo de habilidades informativas". 2008, http://bivir.uacj.mx/dhi/Eventos/Default.htm.

- Séptimo Encuentro Internacional DHI, Tema: "Redes de Colaboración en Programas DHI” 2011, http://bivir.uacj.mx/dhi/Eventos/Default. htm.

$\mathrm{Al}$ analizar las ponencias presentadas en estas siete versiones se encuentra gran coincidencia con los resultados de las universidades-IES identificadas en este análisis de contenidos Web, pues son casi todas las mismas instituciones que ubicamos en niveles 1 o 2 de DHI-ALFIN las que han presentado sus desarrollos, experiencias y conceptualizaciones, por lo que se reitera el llamado urgente a que otras universidades/IES mexicanas se unan a estos procesos de incorporación y de formación en competencias informacionales, teniendo en cuenta la necesidad apremiante de estas competencias en los ciudadanos del siglo XXI.

Por otro lado la labor de gestión y posicionamiento de esta temática que desde México y para Iberoamérica y el mundo ha realizado el profesor-investigador Jesús Lau es admirable, además del trabajo impulsor que desde hace 
varios años ha tenido en la IFLA (International Federation of Library Associations and Institutions) para posicionar la alfabetización informacional (information literacy) como sección, ${ }^{14}$ al coordinar la elaboración de un estado del arte (Lau et al., 2006) y al crear el directorio INFOLIT Global, ${ }^{15}$ y a su vez el trabajo continuo de investigación que ha hecho desde el CUIB (Centro Universitario de Investigaciones Bibliotecológicas) con diferentes proyectos y publicaciones (ahora en acceso abierto ${ }^{16}$ ) relacionados directa e indirectamente con esta temática.

\section{Bibliografía}

ALA/ACRL (2000), Information Literacy Competency Standards for Higher Education, disponible en: www.ala.org/ala/acrl/acrlstandards/informationliteracy competency.htm [20 de septiembre de 2009].

Area-Moreira, M. (2007), Adquisición de competencias en información. Una materia necesaria en la formación universitaria, Documento marco de REBIUN para la CRUE, en REBIUN, disponible en: http://www.rebiun.org/export/docReb/resumenadquisicion41 FF98.doc [24 de agosto de 2010].

Area Moreira, M.; Gros, B. y Marzal, M.A. (2008), Alfabetizaciones y tecnologías de la información y la comunicación, Madrid: Editorial Síntesis, 116 p.

Benito-Morales, F. y Gómez-Hernández, J. A. (2001), "De la formación de usuarios a la alfabetización informacional: Propuestas para enseñar las habilidades de información”, en Scire: Representación y organización del conocimiento, (71), 53-83, disponible en: http://www.um.es/gtiweb/jgomez/publicaciones/ alfabinforzaragoza2.PDF [23 de septiembre de 2007].

Detlor, B. y Lewis, V. (2006), “Academic Library Web Sites: Current Practice and Future Directions", en The Journal of Academic Librarianship, 32 (3): 251-258.

EMPATIC (2009), Report on Current State and Best Practices in Information Literacy, European Commission: Lifelong Learning Programme.

Gardner, S.J; Juricek, J.E. y Xu, F.G. (2008), "An Analysis of Academic Library Web Pages for Faculty”, en The Journal of Academic Librarianship, 34 (1): 16-24. 
Gomes, H.; Prudêncio, D. S. y Conceição, A. V. (2010), "A mediação da informação pelas bibliotecas universitárias: um mapeamento sobre o uso dos dispositivos de comunicação na web", en Informação ES Sociedade: Estudos, 20 (3): 1-12, disponible en: http://www. ies.ufpb.br/ojs2/index.php/ies/article/view/9047/4812 [9 de enero de 2011].

Gómez-Hernández, J. A. (2007), "Alfabetización informacional: cuestiones básicas", en Anuario ThinkEPI, disponible en: http://www. thinkepi.net/alfabetizacion-informacional-cuestiones-basicas [10 de noviembre de 2009].

Gómez Hernández, J. A. y Benito Morales, F. (2001), "De la formación de usuarios a la alfabetización informacional: Propuestas para enseñar las habilidades de información", en Scire: Representación y organización del conocimiento, 7 (2), 53-83, disponible en: http://www.um.es/gtiweb/jgomez/publicaciones/alfabinforzaragoza2.PDF [25 de octubre de 2011].

Gutiérrez, A.; Vidal, F. y Area-Moreira, M. (2012), Alfabetización Digital y Competencias Informacionales, Barcelona: Fundación Telefónica-Fundación Encuentro-Editorial Ariel, 206 p., disponible en: https://ddv.ull.es/users/manarea/public/libro_\%20Alfabeti zacion_digital.pdf [20 de julio de 2012].

Lau, J. et al. (2007), "Information Literancy: An international state-ofthe art report", en INFOLIT GLOBAL, disponible en: <www.uv.mx/ usbi_ver/unesco> [consultado: 9-10-2009].

Pinto-Molina, M. y Uribe-Tirado, A. (2011), "Formación del bibliotecario como alfabetizador informacional”, en: Anuario ThinkEPI, disponible en: http://www.thinkepi.net/anuario-thinkepi/anua rio-thinkepi-2011 [consultado: 29-04-2011].

Pinto-Molina, M.; Sales, D. y Osorio, P. (2008), Biblioteca universitaria, CRAI y alfabetización informacional, Gijón: Ediciones TREA, $246 \mathrm{p}$.

REBIUN-CRUE (2009), Competencias informáticas e informacionales en los estudios de grado, en: REBIUN-CRUE, disponible en: http:// rebiun.org/opencms/ opencms/handle404? exporturi=/export/ docReb/documento_competencias_informaticas.pdf \&\% $\% \mathrm{~d}$ [consultado: 2-10-2011].

Sontang, G. (2011), "Information literacy classification-Colombia”, Facilitating Information Literacy Education, disponible en: http:// www.linkedin.com/groupItem? view $=\&$ gid $=1850160 \&$ type $=$ mem ber\&item $=48506240 \&$ qid $=$ b59f064e-713d-4286-b98d-fe 57233783 9f\&trk=group_most_popular-0-bttl\&goback=\%2Egmp_1850160 [consultado: 27-03-2011].

UNESCO (2003), Declaración de Praga. Hacia una sociedad informacionalmente alfabetizada, disponible en: http://www.eubca.edu.uy/mate riales/servicio_de_informacion_y_consulta/praga.pdf [consultado: 10 de agosto de 2008]. 
UNESCO (2005), Declaración de Alexandría. Faros para la Sociedad de la Información, disponible en: http://archive.ifla.org/III/wsis/Bea conInfSoc-es.html [consultado: 10 de agosto de 2008].

UNESCO (2011), Declaración de Fez. Media and Information Literacy, disponible en: http://www.unesco.org/new/fileadmin/MULTI MEDIA/HQ/CI/CI/pdf/news/Fez\%20Declaration.pdf, [28 de julio de 2011].

Uribe-Tirado, A. (2010a), Avances y perspectivas de ALFIN en Iberoamérica. Una mirada desde la publicación académico-científica y la web 1.0 y 2.0, en: Congreso INFO Cuba 2010 - Instituto de Información Científica y Tecnológica (IDICT), disponible en: http:// eprints.rclis.org/handle/10760/14638 [21 de febrero de 2011]. , (2010b), "La Alfabetización Informacional en Iberoamérica. Una aproximación a su pasado, presente y futuro desde el análisis de la literatura publicada y los recursos web”, en IBERSID Revista de sistemas de información y documentación, Universidad de Zaragoza, 2010: 165-176, disponible en: http://eprints.rclis. org/handle/10760/15060 [21 de febrero de 2011].

(2010c), "La Alfabetización Informacional en la Universidad. Descripción y Categorización según los Niveles de Integración de ALFIN. Caso Universidad de Antioquia”, en Revista Interamericana de Bibliotecología, 33 (1), 10-45, disponible en: http:// aprendeenlinea.udea.edu.co/revistas/index.php/RIB/articleview/ 6280/5801 [21 de febrero de 2011].

(2011), Informe-Estado del Arte de la Alfabetización Informacional en Colombia, en: IFLA-Information Literacy Section, Marzo 2011, 26 p., disponible en: http://www.ifla.org/en/publi cations/information-literacy-state-of-the-art-report-colombia-espa-ol [21 de febrero de 2011].

Webber, S. y Johnston, B. (2006), "Working towards the information literate university”, en Walton, G. y Pope, A. (Eds) Information literacy: recognising the need, Staffordshire University, Stoke-onTrent: 17 May 2006, Oxford: Chandos, pp. 47-58, disponible en: http://dis.shef.ac.uk/sheila/staffs-webber-johnston.pdf, [20 de noviembre de 2009].

White, M.D. y Marsh, E. E. (2006), "Content Analysis: A Flexible Methodology”, en Library Trends, 55 (1): 22-45. 


\title{
Anexo 1
}

\author{
Listado de literatura sobre DHI-ALFIN \\ analizada para el caso mexicano
}

\section{Artículos}

Angulo Marcial, N. (2003), Normas de competencias en información, Bid, (11), http://www.ub.es/bid/11angul2.htm (consultado: 2510-2011).

(2004), "El problema de la información en el contexto de la educación superior”, Forinf@. Revista Iberoamericana de los Usuarios de la Información, (26), 9-18.

(2006), "Pertinencia del término 'alfabetización en información' en el contexto de la bibliotecología superior”, en Revista Brasileira de Biblioteconomia e Documentação: Nova Série, 2(2), 120, http://www.febab.org.br/rbbd/ojs-2.1.1/index.php/rbbd/article/view/15/3 (consultado: 25-10-2011).

Cortés Vera, J. (2005), "El trinomio comunidades de aprendizaje, bibliotecas digitales y competencias informativas", en Biblioteca Universitaria, 8(1), 21-29, http://redalyc.uaemex.mx/pdf/285/28580104.pdf (consultado: 25-10-2011).

(2007), "El desarrollo de competencias informativas en estudiantes universitarios a través de un curso con valor en créditos", en Biblios (29), http://www.scielo.org.pe/pdf/biblios/n29/ a02n29.pdf (consultado: 25-10-2011).

García-Colorado, C.; Irigoyen-Camacho, R. M. y Llorens-Cruset, A. (2008), "Alfabetización informativa: ¿proyecto o quimera? Crítica Bibliotecológica", en Revista de las Ciencias de Información Documental, 1(1), 67-80, http://eprints.rclis.org/bitstream/10760/12 616/1/c.b.vol.1.no.1.garcia-colorado.pdf (consultado: 25-10-2011).

García-Orozco, J.F. (2007), "El desarrollo de competencias formativas en línea, en bibliotecología y ciencias de la información, 2007”, Revista AIBDA. Asociacion Interamerica de Bibliotecarios, Documentalistas y Especialistas en Información Agricola, 28(1), http://eprints.rclis.org/bitstream/10760/10127/1/2007.Garcia-O rozco.J.F.AIBDA.28.1.competencias.pdf (consultado: 25-10-2011).

Gomez Cruz, M. y Montes De Oca, A. R. (2011), "Habilidades informativas en jóvenes universitarios de la UNIVA", en Revista de la Universidad del Valle de Atemajac, 25(71), Sep.-Dic.

Gómez Hernández, J. A. y Licea De Arenas, J. (2011), "Las competencias informacionales y la evaluación de la docencia universitaria", en Revista Universitaria Digital de Ciencias Sociales, 2(1). http:// www.cuautitlan.unam.mx/rudics/ejemplares/0102/pdf/art08.pdf (consultado: 25-10-2011) [Trabajo conjunto: España-México]. 
Gómez Hernández, J. A.; Licea De Arenas, J. y Rodriguez, J. V. (2004), "Information literacy: implications for Mexican and Spanish university students”, en Library Review, 53(9), 451-460, http://www.um.es/ gtiweb/jgomez/publicaciones/alfinmexicoumu.pdf (consultado: 2510-2011), (Trabajo conjunto: España-México).

Gómez Hernández, J. A., Licea De Arenas, J. y Valles Valenzuela, (2009), "Más sobre alfabetización informacional", en Acimed, 20(6), http://scielo.sld.cu/pdf/aci/v20n6/aci071209.pdf (consultado: 25-10-2011), (Trabajo conjunto: España-México).

Gómez Sustaita, M. R., "El modelo educativo basado en competencias en el bachillerato mexicano y el desarrollo de habilidades de información, 2011”, en Infoconexión: Revista Chilena de Bibliotecología, (2), http://eprints.rclis.org/bitstream/10760/15777/1/Infoconexion \%20N\%C2\%B02\%20\%20Gomez\%20Sustaita $\% 20$ Mar\%C3\%ADa.pdf (consultado: 25-10-2011).

Hernández Salazar, P. (2007), "La relación entre los estudios y la formación de usuarios de la información", en Revista General de Información y Documentación, 17(2), 103-121, http://dialnet.unirioja.es/servlet/articulo? codigo $=2550841 \&$ orden $=209785 \&$ info $=$ li nk (consultado: 25-10-2011).

Lau, J. (2001), "Faculty-librarian collaboration: a Mexican experience./Colaboración Docentes Bibliotecarios: una experiencia Mexicana”, en Reference Services Review, 29(2), 95-106.

Lau, J. y Cortés, J. (2009), "Habilidades informativas: convergencia entre ciencias de información y comunicación”, en Comunicar, (32), 21-30, http://www.revistacomunicar.com/verpdf.php? nume ro=32\&articulo=32-2009-06 (consultado: 25-10-2011).

Licea De Arenas, J. (2007), "La evaluación de la alfabetización informacional: principios, metodologías y retos", en Anales de Documentación, (10), 215-232, http://revistas.um.es/analesdoc/ article/ view/1161/1211 (consultado: 25-10-2011).

(2009), "La alfabetización informacional en el entorno hispanoamericano", en Anales de documentación, (12), 93-106, http://revistas.um.es/analesdoc/article/view/70261/67731 (consultado: 25-10-2011).

Moreno Reséndez, A. et al. (2004), "La acreditación de las competencias informacionales como requisito de los programas de maestría y doctorado", en Anales de documentación, (7), 375-396, http://re vistas.um.es/analesdoc/article/view/1631/1681 (consultado: 2510-2011).

Palma Peña, J. M. (2009), "La alfabetización informativa tecnológica: estrategia fundamental en las bibliotecas del Siglo XXI", en Revista Interamericana de Bibliotecología, 32(1), 155-172, http://revinut. udea.edu.co/index.php/RIB/article/viewFile/2756/2218 (consultado: 25-10-2011). 
Romero Esquivel, R. (2007), "Bibliotecas virtuales y alfabetización en información”, en Apertura, 7(6), 92-99, http://www.udgvirtual. udg.mx/apertura/num6/pdfs/bibliotecas.pdf (consultado: 25-102011).

Sánchez Ambriz, G. (2007), "Experiencia de aplicación de servicios de alfabetización informacional para docentes universitarios mayores de 35 años en la UNAM-México”, en Anales de Documentación, (10), 375-396, http://revistas.um.es/analesdoc/article/view/1261/1311 (consultado: 25-10-2011).

Solis Valdespino, B. E. (2005), "Estudio de usuarios en el ámbito universitario Mexicano (Estado del arte 1970 - 2003)", Hemera: Revista de ciencias bibliotecológica y de la Información, Año 3, 3(5): 21-31.

Tarango, J. y Marzal, M. A. (2011), "Funcionalidad de la alfabetización informativa y sus ámbitos de actuación: aproximaciones a los entornos mexicano y español", en Revista Interamericana de Bibliotecología, 34 (3), 283-299, http://aprendeenlinea.udea.edu.co/ revistas/index.php/RIB/article/view/11967/10845, (consultado: 0506-2012), (Trabajo conjunto: México-España).

Valdez Ramos, J; Solis Valdespino, B. E. y Ramírez Campos, M. A. (2006), "La alfabetización informativa en la diversidad: un acercamiento a seis páginas web de bibliotecas universitarias", en Biblioteca Universitaria, 9(22), http://redalyc.uaemex.mx/pdf/285/28590204. pdf (consultado: 25-10-2011).

Valadez Olguin, R. G. et al. (2008), "Diseño de un curso en línea para la alfabetización informacional bajo el modelo ADDIE: una experiencia en la UNAM", IBERSID: revista de sistemas de información y documentación, Universidad de Zaragoza, 2007, 267-274, http:// ibersid.eu/ojs/index.php/ibersid/article/view/3313/3074 (consultado: 25-10-2011).

(2008), "Vinculación entre usuarios, biblioteca y núcleos académicos en el uso de los recursos de información: una propuesta de alfabetismo informacional en la UNAM", IBERSID: revista de sistemas de información y documentación, 223-230.

Valerio-Ureña, G. y Valenzuela-González, R. (2011), "Redes sociales y estudiantes universitarios: Del nativo digital al informívoro saludable", en El Profesional de la Información, 20 (6), 667-670.

Varela Varela, A. (2008), "Sistema informacional, lectura y conocimiento: Gerenciando el flujo de una herramienta cognitiva hacia la alfabetización informacional”, en Investigación Bibliotecológica, 22(44), 89-102.

Vega Díaz, G.; Rojas-Drummond, S. y Mazón Parra, N. (2008), "Apropiación de habilidades para evaluar información: estudio con alumnos de educación primaria”, en Investigación Bibliotecológica, 22 (45), 79-103, http://www.ejournal.unam.mx/ibi/vol22 -45/IBI002204505.pdf (consultado: 25-10-2011). 


\section{Ponencias}

Barriga Ramírez, T. J. y Puente Palazuelos, C. (2012), ALFIN en las Bibliotecas de los Centros de Investigación y Posgrado del IPN: caso de Estudio CIIEMAD, CICIMAR Y CIIDIR-UNIDAD SINALOA, en Seminario "Lecciones aprendidas en programas de alfabetización informacional en Iberoamérica”, Congreso INFO Cuba, https:// docs.google.com/open?id=0BweUYcipCswRalg1NDB4Q0lxeFk (consultado: 19-4-2012).

Cortés, J. (1999), Desarrollo de habilidades informativas en sistemas universitarios: ¿Por qué y para quién?, en Mesa Redonda sobre Formación de Usuarios, XXX Jornadas Mexicanas de Biblioteconomía, Morelia, Mich., http://bivir.uacj.mx/dhi/PublicacionesUACJ/Docs/Ponencias/PDF/PonAMBAC99.pdf (consultado: 2510-2011).

(2003), Competencias informativas y comunidades de aprendizaje: nuevos escenarios para los programas de formación de usuarios, en X Reunión de Bibliotecarios de la Península de Yucatán, Mérida, Yucatán, http://bivir.uacj.mx/dhi/PublicacionesUACJ/Docs/Ponencias/PDF/Ponmerida03.pdf (consultado: 2510-2011).

Cortés, J. y Lau, J. (1999), Information Skills for Life-Long Learning / Habilidades informativas para el aprendizaje de toda la vida, en 65th IFLA Council and General Conference, Bangkok, Thailand, http://bivir.uacj.mx/dhi/PublicacionesUACJ/Docs/Ponencias/ PDF/ponif992.pdf (consultado: 25-10-2011).

Cortés, J.; Mears, B. y Palacios, J. M. (2006), Evolución de los programas de Desarrollo de Habilidades Informativas y las competencias del bibliotecario, en XXXVII Jornadas Mexicanas de Biblioteconomía, Tlaquepaque, Jalisco, http://bivir.uacj.mx/dhi/PublicacionesUACJ/ Docs/Ponencias/Ponencia\%20Jornadas\%202006\%20finaljueves\% 208.pdf (consultado: 25-10-2011).

García Ochoa, R. y Solís Valdespino, B. E. (2008), “Experiencias en el desarrollo del tutorial Sesión Inicial del programa de formación de usuarios de la biblioteca Stephen A. Bastien del CELE-UNAM”, en Memorias del $9^{\circ}$ Congreso Internacional y $12^{\circ}$ Nacional de Material Didáctico Innovador. Nuevas tecnologías, pp.320-324, http:// www.uam.mx/matdidac/imags/Memorias2008.pdf (consultado el 10 de abril de 2010).

González Harmon, R., y Limón González, E.G. (2005), Estudio del perfil, necesidades de información y programa de formación de usuarios: caso Biblioteca de la Facultad de Filosofía y Letras de la $\mathrm{UACH}$, en Foro Transfronterizo de Bibliotecas, Chihuahua (México), 331-344, http://eprints.rclis.org/bitstream/10760/9486/1/200 6.R.GonzalezH.yEG.LimonG.Foro.UACH.pdf (consultado: 2510-2011). 
Gómez Hernández, J. A. y Licea De Arenas, J. (2008), La alfabetización informacional: su reflejo en la formación de los bibliotecólogos y en los servicios de las bibliotecas de universidades públicas de México y España, en VIII Encuentro EDIBCIC (Asociación de Educadores, Investigadores de Bibliotecología, Archivología, Ciencias de la Informa-ción y Documentación de Iberoamérica y el Caribe), México, DF, Noviembre 12-14 [Trabajo conjunto: España-México].

Gómez Sustaita, M.R.E. (2000), La enseñanza de las habilidades informativas, en Documentos, http://bibliotecas.uag.mx/rebco/ DOCUMENTOS/LA\%20ENSE\%C3\%91ANZA\%20DE\%20LAS\%20 HABILIDADES\%20INFORMATIVAS.pdf, (consultado: 25-10-2011).

Hernández Salazar, P. (2011), La alfabetización informativa en México, en VIII Seminario Hispano Mexicano de Investigación en Bibliotecología y Documentación, Madrid.

Ibañez Marmolejo, M. y Solís Valdespino, B. E. (2010), "El desarrollo de competencias para la alfabetización informativa de los recursos electrónicos de la UNAM (la biblioteca del CELE)" en Memorias del $12^{\circ}$ Congreso Internacional y $15^{\circ}$ Nacional de Material Didáctico Innovador, http://www.uam.mx/matdidac/imags/memorias2010.pdf (consultado el 4 de mayo 2012).

Lau, J. (1999), Información: Insumo básico de la educación orientada al aprendizaje, en VI Coloquio de Bibliotecarios, Feria Internacional del Libro, FIL '99, Universidad de Guadalajara, Guadalajara, Jal., México, http://bivir.uacj.mx/dhi/PublicacionesUACJ/Docs/ Ponencias/PDF/ponfil99.pdf (consultado: 25-10-2011).

(2011), Proyectos ALFIN - IFLA y UNESCO, en Primeras Jornadas Nacionales de Alfabetización Informacional (Mendoza-Argentina), http://alfin.uncu.edu.ar/presentaciones/Dia2/Ponencia -JesusLau.pptx (consultado: 1-12-2011).

Mears, B. (2012), "Lecciones aprendidas del programa de Desarrollo de Habilidades", en Seminario Lecciones aprendidas en programas de alfabetización informacional en Iberoamérica, Congreso INFO Cuba, https://docs.google.com/open?id=0BweUYcipCswR ZmJ2dEQ1bEhKWVE (consultado: 19-4-2012).

Mireles, C. y Vázquez, R. (2012), "Prácticas innovadoras sobre Alfabetización Informacional en entornos virtuales. Experiencias en la Biblioteca Virtual CREATIVA de la Universidad Autónoma de San Luis Potosí”, en Seminario Lecciones aprendidas en programas de alfabetización informacional en Iberoamérica, Congreso INFO Cuba, https://docs.google.com/open?id=0BweUYcipCswR Q2VzQXg2MHBudms (consultado: 19-4-2012).

Morales Campos, E., (2006), El acceso a la información, alfabetización informativa y las universidades. que el bibliotecario latinoamericano promueva alfabetización en información, en World Library and Information Congress: 72nd IFLA General Conference and Council, Seoul, Korea, http://archive.ifla.org/IV/ifla72/ papers/129-Campos-es.pdf (consultado: 25-10-2011). 
Ramírez Leyva, E. M. (2002), Lectura, alfabetización en información y cultura de la Información, Documento preparatorio, en Reunión de Expertos en Alfabetización en Información, Praga, http://www.cerlalc.org/redplanes/Documentos/Biblioteca/Nue vas_tecnologias_lectura_alfabetizacion_en_informacion.pdf (consultado: 25-10-2011).

Ríos Morgan, M.B. y Lau, J. (2006), Desarrollo de Habilidades Informativas para el aprendizaje: una propuesta para la escuela de Economía de la UAS, en $5^{\circ}$. Encuentro Internacional DHI, UACJ.

Romo González, J. R. et al. (2012), Cultura informacional en Comunidades de Aprendizaje Universitarias: Brecha Digital por tipo de Educación y Área Académica, en Seminario "Lecciones aprendidas en programas de alfabetización informacional en Iberoamérica”, Congreso INFO Cuba, https://docs.google.com/open?id=0B weUYcipCswRU0dJT0VnZEdXZGM (consultado: 19-4-2012).

Rovalo De Robles, M. L. (2004), Aportes de la dirección general de bibliotecas al desarrollo de habilidades informativas de la UNAM, en III Conferencia Internacional sobre Bibliotecas Universitarias, México, http://dgb.unam.mx/eventos/reunion/conf2004/ROVALO.pdf, (consultado: 25-10-2011).

Solís Valdespino, B. E. y J. Valdez Ramos (2006), "Los recursos de información electrónica de la Biblioteca 'Stephen A. Bastien': un apoyo al Curso de Formación del Profesores del Centro de Enseñanza de Lenguas Extranjeras de la Universidad Nacional Autónoma de México", en Memorias del Encuentro Internacional de Educación Superior UNAM - Virtual Educa 2006, http://e-spacio. uned.es/fez/view.php?pid=bibliuned:1518, (consultado: 25-52012).

Tarango, J.; Pirela M.; J. et al. (2011), Habilidades del pensamiento y habilidades informativas (DHP+DHI): binomio estratégico del currículo de Bibliotecología y Ciencia de la Información, en $7^{\circ}$ Encuentro Internacional DHI, Ciudad Juárez, México, (consultado: 25-10-2011) [Trabajo conjunto: MÉXICO-VENEZUELA].

Tarango, J. y Mendoza-Guillén, G. (2011), Hacia una definición de competencias profesionales en ALFIN: Bibliotecarios y educadores ¿antagónicos o complementarios?, en VIII Seminario Hispano Mexicano de Investigación en Bibliotecología y Documentación, Madrid.

Tiscareño, M.l. y Mears, B. (2002), Propuesta de aplicación de una de las normas de la ACRL en un curso de formación de usuarios, en 3er. Encuentro Internacional de Desarrollo de Habilidades Informativas, Cd. Juárez, Chih., http://bivir.uacj.mx/dhi/PublicacionesUACJ/Docs/Ponencias/PDF/BMears_LTiscare\%C3\%B1o.pdf (consultado: 25-10-2011).

Valadez Olguín, R. G. et al., (2007), "Diseño de un curso en línea para la alfabetización informacional bajo el modelo ADDIE: una experiencia en la UNAM", IBERSID: revista de sistemas de información y documentación. 
Veladez O., R. G. et al. (2011), El uso de las redes sociales: un camino al alfabetismo informacional. Estudio de caso en la Facultad de Estudios Superiores Cuatitlán-UNAM, en VI Congreso internacional de bibliotecas universitarias y unidades de investigación, Universidad de Panamá, Panamá.

Vega Díaz, G. y Quijano, Á. (2010), Comunidades de práctica y alfabetización informacional, Congreso IBERSID, Zaragoza, http:// www.ibersid.eu/ojs/index.php/ibersid/article/view/3878/3598 (consultado: 25-10-2011).

\section{Libros/Capitulos de libro}

Calva González, J. J. (2009), Satisfacción de usuarios: la investigación sobre las necesidades de información, México: UNAM-CUIB. 52 p., http://132.248.242.3/ publica/archivos/libros/satisfaccion_ usuarios.pdf (consultado: 25-10-2011).

(2010), Necesidades de información y comportamiento en la búsqueda de información de los adolescentes, México, UNAM-CUIB. 252p., http://132.248.242.3/ publica/archivos/libros/necesidades_ adolescentes.pdf (consultado: 25-10-2011).

Cortés, J. y Lau, J. (2000), Desarrollo de Habilidades Informativas en Instituciones de Educación Superior, México, UACJ, 132p., http:// bivir.uacj.mx/dhi/PublicacionesUACJ/Docs/Libros/Memorias_ Primer_DHI.pdf (consultado: 25-10-2011).

(2004), Normas de alfabetización informativa para el aprendizaje, México: UACJ, 269 p., http://bivir.uacj.mx/dhi/PublicacionesUACJ/Docs/Libros/Memorias_Tercer_Encuentro_DHI.pdf, (consultado: 25-10-2011).

Cortés, J. y Mears, B. (2006), Implementación de programas de alfabetización informativa: contextos y experiencias, México: UACJ, 265 p.

Gómez Hernández, J. A. y Licea De Arenas, J. (2005), “El compromiso de las bibliotecas con el aprendizaje permanente. La Alfabetización Informacional”, en López López, P. y Gimeno Perelló, J. (eds.) Información, conocimiento y bibliotecas en el marco de la globalización neoliberal, Gijón: Trea, 145-179, http://www.um.es/ gtiweb/jgomez/publicaciones/aprendizajepermanentebibliotecas. pdf, (consultado: 25-10-2011), [Trabajo conjunto: España-México].

Gómez Sustaita, M. R. E. (2009), Educación de usuarios de la información: elaboración de programas para el desarrollo de babilidades informativas, México, Infoconsultores, $144 \mathrm{p}$.

Hernández Salazar, P. (1998), La formación de usuarios de la información en instituciones de educación superior, México: CUIB, 76 p.

(2004), Modelo para generar programas sobre la formación en el uso de tecnologías de información, México: UNAM, Centro Universitario de Investigaciones Bibliotecológicas, 108 p. 
Hernández Salazar, P. coord. (2008), Métodos cualitativos para estudiar a los usuarios de la información, México: UNAM, Centro Universitario de Investigaciones Bibliotecológicas, http://iibi.unam. mx/opLibros.html (consultado 25-05-2012).

Lau, J. y Cortés, J. (2000), La Instrucción de Usuarios ante los Nuevos Modelos Educativos, México: UACJ, 2000, 132p., http://bivir.uacj. $\mathrm{mx} / \mathrm{dhi} /$ PublicacionesUACJ/Docs/Libros/Memorias_Segundo_ DHI.pdf, (consultado: 25-10-2011).

Lau, J. (2006), Information Literacy: International Experiences, Munich, Alemania: K. G. Saur. (2006), Guidelines on Information Literacy for Lifelong Learning, Munich, Alemania: K.G. Saur, http://archive.ifla.org/ VII/s42/pub/IL-Guidelines2006.pdf (consultado: 25-10-2011).

Lau, J. et al. (2007), Information Literacy: An international state-of-the art report, UNESCO-IFLA, http://www.infolitglobal.info/media/ UNESCO_IL_state_of_the_art_report_-_Draft070803.doc (consultado: octubre 30 de 2009).

Licea De Arenas, J. y Arenas Vargas, M. (2011), Manual del investigador novel, México: Universidad Autónoma Metropolitana-Xochimilco, $222 \mathrm{p}$.

Rivera, M (2000), "Madrid: manejo de recursos informativos para docentes”, en Lau, J. y Cortés, J (Eds), Praxis: Experiencias del Área de Apoyo Académico, UACJ, Juárez, http://bivir.uacj.mx/dhi/ PublicacionesUACJ/Docs/Articulos/madrid.pdf, (consultado: 2510-2011).

Solis Valdespino, B. E. y Ramirez Campos, M. A. (2010), "Manual de ejercicios de para el taller", Taller sobre el uso de recursos electrónicos de información para los estudiantes del posgrado en lingüística de la UNAM, México, UNAM, CELE, Biblioteca "Stephen A. Bastien: Programa de Formación de Usuarios, http://www.cele.unam. $\mathrm{mx} /$ biblioteca/pdfs/Manual\%2010\%20v2.1.pdf (consultado: 2505-2012).

Valdez Ramos, J. y Solis Valdespino, B. E. (2009), “El programa de formación de usuarios en una biblioteca universitaria: un proyecto interdisciplinario", en Memorias del Simposio La Psicología y la Educación a Distancia, México: UNAM, Facultad de Psicología y la Coordinación de La Universidad Abierta y Educación a Distancia, http://www.ead.psicol.unam.mx/memoria2009/ (consultado: 10-05-2012).

Valdez Ramos, J., Solís Valdespino, B. E. y Ramirez Campos, M. A. (2007), Manual básico para la búsqueda y recuperación de información, México, UNAM, CELE, 1 CD-Rom. 


\section{Trabajos de grado (pregrado)}

Campos Hernández, L. (2007), Curso taller para la búsqueda y recuperación de información en bibliotecas de los Institutos Nacionales de Salud, Trabajo de grado, Bibliotecología, Escuela Nacional de Biblioteconomía y Archivonomía, http://www.campusvirtualhgm.net/seminario/sites/default/files/CursoTallerBusquedaRecuparacionINNSZ.pdf (consultado: 25-10-2011).

Morales Sánchez, Y. y Venegas Valencia, A. L. (2011), Alfabetización informacional en adultos mayores ¿Cómo interrelacionarlos dentro de una biblioteca pública?, Trabajo de grado, Bibliotecología. Escuela Nacional de Bibliotecología y Archivística, http://eprints. rclis.org/bitstream/10760/15873/1/ALFABETIZACION\%20INFORMA CIONAL\%20EN\%20ADULTOS\%20MAYORES.pdf (consultado: 2510-2011).

\section{Tesis (posgrado)}

Ángulo Marcial, N. (2003), El concepto de alfabetismo en información en el contexto de la bibliotecología latinoamericana, Tesis de doctorado en Bibliotecología y Estudios de la Información, Facultad de Filosofía y Letras, Universidad Nacional Autónoma de México (UNAM), México, DF.

Cortés, J. (2002), Construcción de normas para evaluar competencias informativas en los estudiantes universitarios mexicanos, Tesis de doctorado en Bibliotecología y Estudios de la Información, Facultad de Filosofía y Letras, Universidad Nacional Autónoma de México (UNAM), México, DF.

Cortés Gómez, C. F. (2005), Formación de usuarios y modelo educativo: propuesta metodológica para su integración vinculación en instituciones de educación superior, Tesis de Maestría en Bibliotecología y Estudios de la Información, UNAM- Facultad de Filosofía y Letras (consultado: 25-10-2011).

Díaz Velásquez, D. A. (2010), Procesos de alfabetización informacional y construcción del conocimiento en procesos de formación en investigación educativa a través de ambientes a distancia, Tesis de Maestría en Tecnología Educativa, Tecnológico de Monterrey-Escuela de Graduados en Educación, http://catedra.ruv.itesm.mx/bits tream/987654321/394/1/Diaz\%20Demetrio\%201.pdf (consultado: 4-1-2012).

Escudero Amaro, S. G. (2007), Integración del modelo de desarrollo de babilidades informativas: Caso UACH, Tesis de Maestría en Bibliotecología y Ciencias de la información, Universidad Autónoma de Chihuahua, http://fterrazas.files.wordpress.com/2008/02/integra cion.pdf (consultado: 25-10-2011). 
Flores Bañuelos, T. R. (2005), La gestión de la información como alfabetización en la formación de usuarios de la UAM unidad Xocbimilco, Tesis de Maestría en Bibliotecología y Ciencias de la información, Universidad Autónoma Metropolitana Unidad Xochimilco, http:// fterrazas.files.wordpress.com/2008/02/integracion.pdf (consultado: 25-10-2011).

Gloria Gómez, A. L. (2010), Programas de desarrollo de babilidades informativas en los sistemas bibliotecarios de las universidades públicas del estado de San Luis Potosí, Tesis de Maestría en Bibliotecología y Estudios de la Información, UNAM- Facultad de Filosofía y Letras.

Hernández Salazar, P. (2001), Modelo de programas de formación de usuarios de tecnologías de información en instituciones de educación superior, Tesis (Doctorado en Ciencias de la Información), Universidad Complutense de Madrid, Facultad de Ciencias de la Información, Departamento de Biblioteconomía y Documentación (consultado: 25-10-2011).

Lloret Rivas, A. A. (2012), Competencias informacionales, documentales y comunicativas para el desarrollo profesional continuo en medicina: el caso de la Universidad Autónoma Metropolitana Xochimilco de México, Tesis Doctoral en Biblioteconomía y Documentación, Universidad de Murcia (España), http://digitum.um.es/xmlui/ bitstream/10201/27664/1/AlejandroLloretRivas.pdf (consultado: 1-6-2012).

Medina Campoy, S. (2008), La enseñanza de habilidades informativas a través de un programa de formación de usuarios bajo una perspectiva constructivista, Tesis de Maestría en Bibliotecología y Estudios de la Información, UNAM, Facultad de Filosofía y Letras (consultado: 25-10-2011).

Palacios Salinas, C. (2003), Las estrategias de estudio y las habilidades en el uso de la información en estudiantes de la Universidad Anábuac, Tesis de Maestría en Bibliotecología, Universidad Anáhuac, Huixquilucan, México.

Ramírez Carvajal, J. E. (2007), La formación de usuarios de la información en México: desarrollo bistórico, Tesis de Maestría en Bibliotecología y Estudios de la Información, UNAM- Facultad de Filosofía y Letras (consultado: 25-10-2011).

Ríos Morgan, M. B. (2002), Habilidades informativas de los estudiantes de economía de la Universidad Autónoma de Sinaloa: evaluación y propuesta de solución, Tesis de Maestría en Bibliotecología. Universidad Autónoma de Nuevo León.

Salgado Escobar, G. (2009), La cultura informacional en la UPIICSA, Tesis de Maestría en Ciencias en Administración y Desarrollo de la Educación. Instituto Politécnico Nacional, http://itzamna. bnct.ipn.mx:8080/dspace/bitstream/123456789/10455/1/Salga do\%20Escobar\%20Graciela.pdf (consultado: 19-07-2012). 
Solís Valdespino, B. E. (2006), La formación de usuarios en la educación bibliotecológica mexicana: nivel licenciatura, Tesis de Maestría en Bibliotecología y Estudios de la Información, UNAM- Facultad de Filosofía y Letras (consultado: 25-10-2011).

Vega Díaz, M. G. (2003), Formación de Usuarios de la Información en Instituciones de Educación Superior: un Enfoque Sistemático, Tesis de Maestría en Bibliotecología y Estudios de la Información, UNAM- Facultad de Filosofía y Letras.

Vergara Mendoza, G. (2008), Tecnología educativa y desarrollo de babilidades de información: programa de formación de usuarios de la Biblioteca Francisco Xavier Genoveva, Tesis de Maestría en Investigación y Desarrollo de la Educación, Universidad Iberoamericana, México, http://www.bib.uia.mx/tesis/pdf/014986/014986.pdf (consultado: 25-10-2011). 
